\title{
Spontaneous rupture of the spleen - a rare and potentially fatal surgical emergency in the second trimester: Report of a case
}

\author{
M Heetun, MRCS; R Parameswaran, FRCS; K Jamil, FRCPath \\ St Mary's Hospital NHS Trust, Isle of Wight, UK
}

Corresponding author: $M$ Heetun (heetun@hotmail.com)

Spontaneous (non-traumatic) rupture of the normal spleen in pregnancy is a rare clinical event that can endanger the lives of both mother and baby. The aetiology of the condition is unknown, but it occurs most commonly in multiple pregnancies and in the third trimester. We present a case of a spontaneous splenic rupture of a normal spleen at 14 weeks' gestation. Following prompt and aggressive surgical intervention, both mother and baby survived.

S Afr J Obstet Gynaecol 2015;21(2):50-51. DOI:10.7196.SAJOG.858

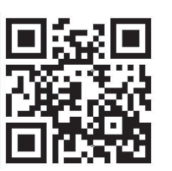

\section{Case report}

A 27-year-old woman at 14 weeks' gestation and with one previous normal delivery presented to the emergency department with a history of sudden onset of severe generalised abdominal pain and collapse. She had no previous significant past medical history. An ultrasound scan 1 month previously had confirmed a viable intrauterine pregnancy.

Initial observations revealed hypotension (77/40 $\mathrm{mmHg})$ and tachycardia (112 bpm). Physical examination revealed diffuse abdominal tenderness, rebound and guarding. Bowel sounds were absent. Preliminary blood tests showed a haemoglobin concentration of $8.7 \mathrm{~g} / \mathrm{dL}$ and a normal coagulation profile. An ultrasound scan performed in the emergency department further confirmed an intrauterine pregnancy, but revealed the presence of free fluid within the pelvis. However, no obvious source for this free fluid could be identified. Aggressive fluid resuscitation was initiated, and the immediate decision was taken to proceed to a laparotomy with a combined surgical and obstetric team.

A large exploratory midline incision was performed. On entry into the abdominal cavity, approximately 3 litres of blood were evacuated. Active bleeding was seen at the splenic hilum, consistent with a splenic rupture. Large abdominal packs were left in the abdominal cavity to apply pressure to the spleen. On further inspection, the uterus, ovaries, fallopian tubes and other

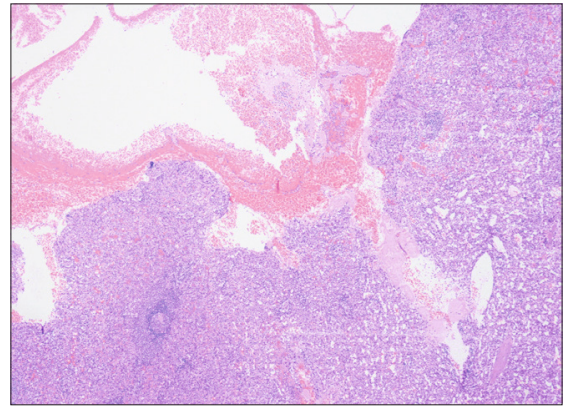

Fig. 1. Rupture of the splenic capsule was confirmed on histological examination of the spleeen.

abdominal organs were intact. Splenectomy was performed by the general surgeon and a non-suction drain left in the subphrenic space. The patient received a total of seven units of blood and five units of fresh-frozen plasma. She made a good recovery in the intensive care unit and auscultation revealed normal fetal heart sounds. She was subsequently transferred to a general surgical ward where her postoperative course was unremarkable and she was discharged 7 days later. She received the postsplenectomy triple vaccine. Histological examination of the spleen confirmed rupture of the splenic capsule (Fig. 1). At the time of writing, her pregnancy had continued without complication.

\section{Discussion}

Spontaneous rupture of the spleen is rare in the absence of trauma and in the nonpregnant female. It is most commonly caused by pre-existing splenic pathology such as a splenic artery aneurysm or an infective cause such as infectious mononucleosis, malaria and typhoid. Orloff and Peskin ${ }^{[1]}$ devised the following criteria for spontaneous splenic rupture in the context of a normal spleen: no history of trauma; the absence of systemic disease affecting the spleen; the absence of perisplenic adhesions to suggest trauma; and the presence of macroscopically and histologically normal splenic parenchyma, vasculature and capsule.

Spontaneous rupture of the normal spleen in pregnancy is also a rare clinical event, presenting as an acute surgical emergency with high maternal and fetal mortality. The diagnosis can be challenging as it can mimic other conditions such as ruptured ectopic pregnancy, placental abruption and uterine rupture. The most common clinical presentation includes left-sided abdominal pain, which can often radiate to the patient's shoulder. If left untreated, the pain becomes more generalised, leading to distension, guarding and rigidity. Haemorrhagic shock then follows, with a reported maternal mortality rate of approximately $70 \%,{ }^{[2]}$ necessitating surgical intervention. In only a few specific cases, especially in Afro-Caribbean patients, splenic preservation is a possible option to avoid diminished immunological competence. However, a low threshold for surgical intervention is still required in these patients. ${ }^{[3]}$ 
The aetiology of splenic rupture in pregnancy remains unknown. It has been reported to be more common in multiple pregnancies and in the third trimester. Wang et al. ${ }^{[4]}$ postulate a number of mechanisms for its cause. Minimal trauma, such as straining for a bowel movement or coughing and sneezing, acts as the inciting event through an increase in intra-abdominal pressure and its transmission to a number of abdominal organs including the spleen. The increase in the circulating blood volume in pregnancy and the reduced space in the peritoneal cavity as a result of the expansion of the gravid uterus may make the spleen more fragile, and therefore more susceptible to rupture. They also suggest that circulating hormones such as oestrogen and progesterone cause structural changes to the spleen in pregnancy that possibly increase the risk of rupture, even after minor trauma. ${ }^{[4]}$

We detailed a case of spontaneous rupture of a normal spleen in a pregnant woman at 14 weeks' gestation. This led to haemorrhagic shock necessitating emergency surgery. Our case fitted the criteria stipulated by Orloff and Peskin ${ }^{[1]}$ in respect of diagnosis of spontaneous rupture of a normal spleen. However, it was unique in that it occurred in the second trimester of pregnancy rather than the third, when the majority of cases occur. To the best of our knowledge, no cases of spontaneous rupture of a normal spleen during the second trimester of pregnancy have been reported in the English literature. If splenic rupture is suspected, an exploratory laparotomy (via a midline vertical incision to maximise access and exposure) should be performed in the vast majority of patients to stop the bleeding. This case highlights the fact that this rare and enigmatic condition can occur at any time during pregnancy and that prompt and aggressive surgical intervention is paramount to saving the lives of both mother and baby.

\section{Learning points}

- Spontaneous rupture of the spleen is a life-threatening clinical event with high maternal and fetal mortality.

- Its aetiology is unknown, but it is more common in the third trimester and in multiple pregnancies.

- It can mimic other acute conditions such as ruptured ectopic pregnancy, uterine rupture and placental abruption.

- In the overwhelming majority of patients, aggressive surgical intervention is indicated to stop the bleeding.

1. Orloff MJ, Peskin GW. Spontaneous rupture of the normal spleen: A surgical enigma. Int Abstr Surg 1958;106(1):1-11.

2. Tanchev S, Popova M, Slalov I. The 'splenic emergency syndrome' during pregnancy (a report of 2 cases). Akush Ginekol (Sofia) 1992;31(1):32-34.

3. Fletcher H, Frederick J, Barned H, Lizarraga V. Spontaneous rupture of the spleen in pregnancy with splenic conservation. West Indian Med J 1989;38(2):114-115.

4. Wang C, Tu X, Li S, Luo G, Norwitz ER. Spontaneous rupture of the spleen: A rare but serious case of acute abdominal pain in pregnancy. J Emerg Med 2011;41(5):503-506. [http://dx.doi. org/10.1016/j.jemermed.2010.05.075] 